\title{
La costa E-O de Andalucía. Discusión sobre su significado geológico
}

\author{
C. Sanz de Galdeano \\ Instituto Andaluz de Ciencias de laTierra (CSIC-Univ. Granada), Facultad de Ciencias, 18071, Granada. \\ csanz@ugr.es
}

RESUMEN

La costa andaluza desde Cabo de Gata a Málaga tiene en su conjunto una dirección E-O. A lo largo de ella, las unidades tectónicas se disponen oblicuamente, en general aflorando las inferiores en la parte oriental y las superiores en la occidental. Dado que la plataforma continental es estrecha, esta oblicuidad no se puede recuperar antes del talud, lo que sugiere la existencia de una falla a lo largo del mismo. En apoyo de esta interpretación hay que señalar la existencia de importantes fallas E-O dextrorsas en la parte más occidental de la Zona Interna Bética (por tanto paralelas a la costa E-O). Estas a su vez forman parte del conjunto de fallas E-O que cortaron la Zona Interna facilitando su traslación hacia el O, traslación tanto mayor cuanto más al S se encuentran los sectores desplazados por las fallas. Estos desplazamientos al O estuvieron ligados a la apertura del mar de Alborán, debido a los empujes que produjo en su borde N, en el lado Bético (por el sur, en el Rif, también produjo desplazamientos). Posteriormente se formaron antiformes E-O importantes y fue la erosión del flanco S del antiforme de la costa lo que produjo el escarpe existente.

Palabras clave: Falla, Mar de Alborán, Tectónica, Zona Interna Bética.

\section{The Andalusian E-W coast. Discussion on its geological significance}

\begin{abstract}
The Andalusian coast from the Cabo de Gata to Malaga has as a whole an E-W direction. The tectonic units are arranged obliquely along it, in general with the lower ones outcropping the in the eastern part and the upper ones in the western part. Since the continental shelf is narrow, this obliquity cannot be recovered before the slope, which suggests the existence of a fault along the slope. In support of this interpretation, it should be noted that in the westernmost part of the Betic Internal Zone there are significant dextral E-W faults (i.e. parallel to the E-W coast), which are some of the E-W faults cutting up the Internal Zone facilitating its westwards translation, greater in the southern most sectors displaced by the faults. These westwards displacements were linked to the opening of the Alboran Sea, due to the pressure that this produced especially on its northern border (on the other hand, deformations also occurred on the Rifian border). Later, important E-O antiforms were formed and the erosion of the $S$ flank of the antiform situated directly to the $N$ of the coast produced the existing scarp.
\end{abstract}

Keywords: Alboran Sea, Betic Internal Zone, fault, tectonics. 


\section{ABRIDGED ENGLISH VERSION}

\section{Introduction}

Land relief never occurs by chance, there is always an underlying cause. This paper is dedicated to looking for the cause which forms the Andalusian E-W coast. In this coast the Alpujarride and Malaguide complexes of the Betic-Rifian Internal Zone crop out (Fig. 1), as well as the tectonic units of the Campo de Gibraltar and locally Neogene and Quaternary sediments.

The structuring of the Betic Cordillera occurred mainly during the Oligocene and early Miocene. Then, the Algerian basin of which the Alboran Sea forms its western end immediately began to open up. A remarkable extension in this opening occurred, which in its turn produced compression at its edges. This provoked the Betic-Rifian Internal Zone to be displaced to the W, and helping that drift important E-W dextral faults were created e.g. those of the Alpujarran corridor (Sanz de Galdeano et al., 1985) (Figs. 1, 2 and 3). Immediately after, from the late Miocene, the region underwent a NNW-SSE compression and formed new faults whose directions are NW-SE and NE-SW to NNE-SSW, as is the Carboneras fault which passes to the Alboran Sea (Larouzière et al., 1988).

As a background, there are previous descriptions of uplift and subsidence of the coast and of the Neogene and Quaternary sediments, but none deals with the subject of this paper. Only Sanz de Galdeano (1983 and 1990) and Ballesteros et al. (2008) have indicated the possible existence of a fault along this coast, but without giving any details on the matter.

\section{Description of the E-W coast of Andalusia}

In the eastern part, the Cabo de Gata, (for the place names see particularly Fig. $2 \mathrm{C}$ and also the Figs. 3 and 4) there are sediments and volcanic rocks from the middle and upper Miocene, formed later than the opening of the Alboran Sea. In the Gulf of Almeria the sediments filling it also consist of upper Miocene to Quaternary deposits.

To the west, from Almería to Aguadulce the coast is formed by Triassic carbonates of the Gador unit la lower Alpujárride unit). There are scarps on the sea of $100-150 \mathrm{~m}$ in height. More to the $W$ the scarp extends approximately $36 \mathrm{~km}$, slightly displaced to the $N$, which does not limit with the sea but to an emerged almost flat area, called the Campo de Dalías. In this scarp the Gador unit presents its Triassic lower terms to the $E$ and the upper ones to the $W$. Above, there is a thrusting unit and, unconformably disposed, there are also upper Miocene sediments. There are also volcanic rocks and andesites, (Baena and Ewert, 1983), which seem to be distributed in E-W direction. The Campo de Dalías area is mainly formed by Pliocene and Quaternary sediments. It is affected by WNW - ESE faults (Baena and Ewert, 1983) and its western side corresponds to a NNW-SSE fault (Marín Lechado, 2005).

For Rodríguez Fernández and Martín Penela (1993) this escarpment coincides with a major fault, but for Marín Lechado (2005) it does not exist as gravimetric data indicate the continuity of the Triassic basement.

More to the $W$ the escarpment again limits with the sea, with values reaching heights of over $300 \mathrm{~m}$. As far as Velez Malaga (about $30 \mathrm{~km}$ E from Malaga) the coast is formed by middle and upper Alpujarride tectonic units. This coast is not completely straight, advancing towards the $S$ in those points in which marbles crop out being more resistant to erosion.

From Vélez Málaga to the $W$, the rocks appearing on the coast belong to Malaguide Complex, giving a somewhat lower relief, as these rocks are less resistant to erosion.

The escarpment continues inland for about $32 \mathrm{~km}$ with the same E-W strike (Fig. 4). Its northern border is formed by the Malaguide Complex, whilst in the southern border late Miocene to Quaternary sediments and units of the Campo de Gibraltar (Flysch Units) appear. This is the westernmost area of the Betic Internal Zone and there are important E-W dextral faults in it (the Albornoque fault, the Coin-Cartama fault, this latter in coalescence towards the $E$ with the position of the described escarpment). There are also important $E$-W dextral faults in this western area, in its northern part. They are situated in the contact between the Internal and External Zones (Fig. 4). These faults are the prolongation of others observed more to the $E$ (Sanz de Galdeano et al., 1985) (Figs. 2 and 3). 
In the submerged part of this E-W coast, the marine platform is narrow, usually on the order of 6-8km. The Pliocene and Quaternary sediments there have been studied (Hernández Molina et al. 2002), but the meaning of the slope, if there is a fault or not, is not clear. Only Ballesteros et al. (2008) indicate the existence of a great dextral transcurrent fault at the bottom of the slope, the latter cut by transversal faults (Fig. 1 B). These authors adapt the fault all along the slope, including the coast between Fuengirola and Torremolinos which is in NNE-SSW direction and also the area surrounding the Campo de Dalías. This last area "should" be a sunken zone, but the faults there existing have uplifted it.

\section{Discussion and conclusions}

The analysis of the tectonic units existing along the coast shows that they appear obliquely, i.e., a certain tectonic position is not maintained. In addition, its general distribution indicates that the units located in upper tectonic position are situated towards the $W$, whereas the lower ones are in the eastern parts. That is, in the western part, up to the proximity of Vélez Málaga, the Malaguide Complex crops out. To the $E$ the upper units of the Alpujárride Complex first appear (Fig. 1 B), and as the complex progresses towards Almeria, the middle and lower Alpujarride units appear.

This shows that the Betic Cordillera is as a whole more uplifted eastwards and more sunken to the W. This is consistent with the interpretation that when the Alboran Sea opened up, in addition to pushing the Internal Zone, it also uplifted its borders more to its eastern part.

The E-W dextral faults cited in the western part of the Betic Internal Zone, displaced its southern sectors to $W$ (Figs. 2, 3 and 4). This segmentation is not a local phenomenon, that is, all the Betic Internal Zone is divided into sectors limited by dextral faults located in E-W corridors (e. g., from $N$ to $S$, the corridor of the Almanzora, that existing between the Sierras Nevada and Filabres, and that of the Alpujarras). There are transcurrent dextral contacts of approximate $N 70^{\circ} \mathrm{E}$ strike and some segments of E-W direction also in the contact between the External and Internal Zones, in which there are dextral displacements. To these features the $E-W$ coast with a similar orientation can be added.

But in the Sierra de Gádor - Campo de Dalías sector, Marín Lechado (2005) indicated that gravimetric data shows the continuity of the Alpujarride basement on both sides of the scarp. However this does not necessarily imply the absence of a fault, since lateral displacements cannot be deduced by gravimetry, and moreover the escarpment is somewhat displaced to the $N$. That is to say, the fault can be placed more to the $S$. In favour of the existence of the fault is the oblique cut of the lithological formations of the Gador unit and the presence of the andesites which would need at least one fissure to extrude.

On the other hand, the generally narrow width of the marine platform does not give enough space to absorb the obliquity of the tectonic units. To explain this, the easiest interpretation is the existence of a fault at the edge of the platform. The fault proposed by Ballesteros et al. (2008) fits very well in its central part with the present interpretation.

This interpretation fits with the opening process of the Alboran Sea which affected the periphery when it extended. On the whole, the Betic-Rifian Internal Zone, moreover its part at present situated in the Alboran Sea, was progressively expelled, to the SW in the Rif and to the W and WNW in the Betics. In this expulsion the Betic Internal Zone was segmented by dextral E-W faults (Fig. 3) and in this process of segmentation the proposed E-W fault of the south coast of Iberia would have been formed. The transversal faults cutting the E-W coast, amongst which the Carboneras fault stands out, were formed later, at the end of the middle Miocene. Also from this time, and particularly from the upper Miocene, important antiforms were formed such as those situated to the $N$ of the E-W coast. The current escarpment began to form on its southern flank, progressively eroding northwards. 


\section{Introducción}

El relieve terrestre nunca se produce de forma aleatoria sino que siempre responde a algunas causas. Estas a veces son evidentes, por ejemplo un relieve en cuesta o la disposición más o menos cónica de un volcán; sin embargo, hay casos en que sus controles no son tan evidentes, aunque sin duda existen.

Este es el caso de la costa andaluza que desde Almería, casi desde Cabo de Gata, llega a Málaga, con una dirección general E-O (Figs. 1 a 3), aunque con algunas irregularidades, presentando a la vez importantes escarpes en muchos puntos. Estos escarpes se prolongan tierra adentro en la cuenca de Málaga.

Al análisis de las posibles causas de este relieve se dedica este artículo.

\section{Situación geológica}

La costa que va desde Almería a la bahía de Málaga corresponde por entero a la Cordillera Bética y dentro de esta a la Zona Interna Bética (Fig. 1 A y B). En ella afloran unidades tectónicas del Complejo Alpujárride y del Complejo Maláguide (cabalgante sobre el anterior). El complejo situado en posición inferior, el Nevado-Filábride, no aflora a lo largo de esta costa. Ligada al Maláguide se encuentra la Dorsal, generalmente separada tectónicamente de ese complejo, pero que a la vez forma parte de su cobertera mesozoica y terciaria.

En la Cordillera Bética, además de la Zona Interna y de la Externa (esta no presente a lo largo de la costa E-O objeto de estudio), hay que citar a las unidades de los Flyschs (unidades del Campo de Gibraltar) superpuestas a ambas zonas y presentes localmente en la cuenca de Málaga. En esta costa también hay algunos afloramientos de sedimentos neógenos, incluso con algunas rocas volcánicas, estas situadas en su parte oriental.

La estructuración de la Zona Interna en complejos y unidades tectónicas cabalgantes se produjo fundamentalmente durante el Oligoceno y parte del Mioceno inferior (Durand-Delga y Fontboté, 1980). Prácticamente a continuación ocurrió en el Mediterráneo occidental la apertura de la cuenca Argelo-Provenzal (Boillot et al., 1984) que hacia el O se prolongó al actual mar de Alborán. De esta manera, el interior del mar de Alborán fue sometido a una gran extensión que hizo que su corteza continental fuera notablemente adelgazada. Esto ocasionó empujes en sus bordes y como consecuencia la Zona Interna fue progresivamente expulsada hacia el $\mathrm{O}$, hacia el SO en el Rif. Esta expulsión fue facilitada con la formación de importantes fallas E-O en la Zona Interna Bética (Sanz de Galdeano, 1990), así las fallas del Corredor de las
Alpujarras, situado al $\mathrm{N}$ de la costa $\mathrm{E}-\mathrm{O}$, y que pasa por el borde S de Sierra Nevada (Sanz de Galdeano et al., 1985) (Figs. 1, 2 y 3).

La deriva hacia el $\mathrm{O}$ de la Zona Interna perdió progresivamente importancia y a partir de finales del Mioceno medio, y sobre todo en el superior, la región sufrió una compresión de dirección NNO-SSE, combinada con una extensión perpendicular. Esto produjo la formación de nuevas fallas de direcciones NO-SE, junto a otras NNE-SSO y NE-SO, algunas de gran importancia como la falla de Carboneras que desde el SE de Almería pasa al mar de Alborán (Larouzière et al., 1988). También durante esta etapa se formaron pliegues E-O, como el gran antiforme de Sierra Nevada.

\section{Antecedentes}

La estructura, estratigrafía y sismicidad del mar de Alborán ha sido estudiada en numerosos artículos, así Woerd et al. (2014), Grevemeyer et al. (2015), Gómez-Peña et al. (2016) y Perea et al. (2018), entre otros muchos. También hay numerosas descripciones de levantamientos y hundimientos de las costas de alrededor del mar de Alborán (Groupe de Recherche Néotectonique, 1977; Zazo et al., 2003; El Kadiri et al., 2010), y acerca de la estratigrafía de los materiales neógenos y cuaternarios actualmente emergidos en la costa o cerca de la costa (Lario et al., 1993; Guerra Merchán et al., 2004) y de los situados en el mar cerca de la costa (Hernández Molina et al., 2002). Pero no conocemos ningún trabajo que intente interpretar con cierto detenimiento las causas de la orientación y características morfoestructurales de la citada costa. A lo largo de la misma, desde Almería a Málaga, Sanz de Galdeano (1983 y 1990) señaló la posible existencia de una falla dextrorsa, pero no entró en ningún detalle. Igual sucede con Sanz de Galdeano (2008 a y 2012). Por su parte, Ballesteros et al. (2008) interpretan que esta costa, incluyendo sectores situados más al $\mathrm{E}$ y al $\mathrm{O}$ también corresponde a una falla dextrorsa, pero tampoco presentan justificaciones a la interpretación.

\section{Análisis de la costa E-O desde Almería a Málaga y su prolongación al 0}

La descripción se hace de $\mathrm{E}$ a O. En ella se describen las formas del relieve junto con los rasgos geológicos existentes. Algunos datos marinos se incluyen más adelante.

En la parte más oriental, Cabo de Gata (muchos de los topónimos citados se encuentran en la Fig. 2 C), la costa se prolonga hacia el $\mathrm{S}$ formando un saliente importante. Afloran allí rocas volcánicas (Pineda 


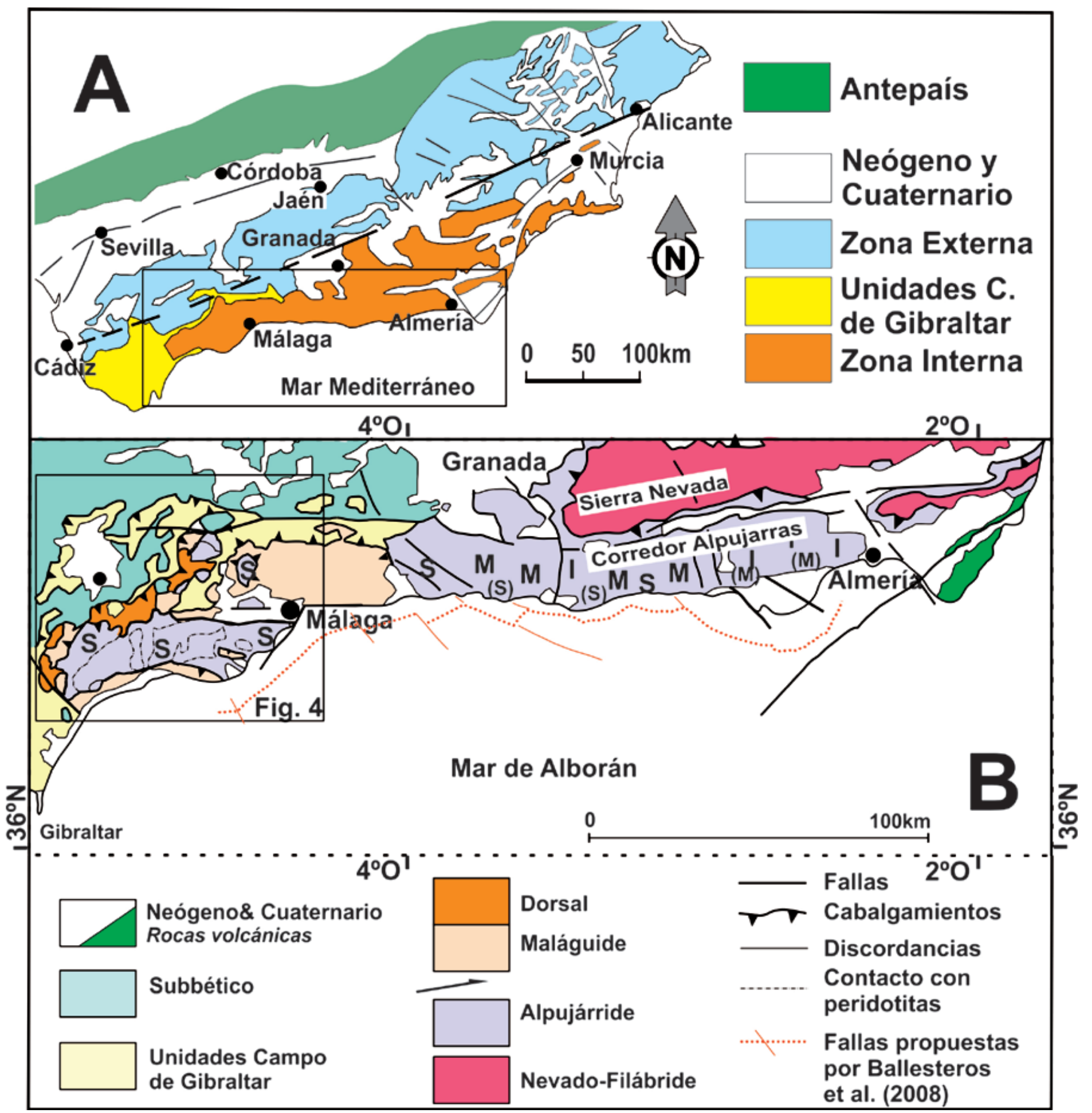

Figura 1. Situación general. A: Posición de la costa E-O en relación con la Cordillera Bética y el mar de Alborán. El rectángulo marca la posición de B. B: Datos generales de los complejos y unidades tectónicas que existen a lo largo de esta costa. Las letras I, M y S indican los tipos de unidades alpujárrides. Las inferiores son señaladas con I, las medias con M y las superiores con S. EI recuadro indica la posición de la Fig. 4.

Figure 1. General setting. A: Position of the E-O coast in relation to the Betic Cordillera and the Alboran Sea. The rectangle marks the position of B. B: General data of the complexes and tectonic units present along the coast. The letters I, $M$ and $S$ indicate the types of the Alpujarride units. The lower ones are marked by $I$, the middle ones by $M$, and the upper ones by S. The inset indicates the position of Figure 4. 
et al., 1981) formadas durante el Mioceno medio y el superior, sobre todo en el superior. Entre muchas de ellas se intercalan rocas sedimentarias marinas, especialmente del Mioceno superior (Serrano, 1992). Por tanto, el Cabo de Gata con sus rocas volcánicas y sedimentarias y la gran falla de Carboneras de dirección NNE-SSO corresponde a rasgos no existentes durante el Mioceno inferior y parte del medio.

El golfo de Almería tampoco es una costa antigua. Está formado por materiales del Neógeno superiory, sobre todo, cuaternarios. Estos, en buena parte, son rellenos aportados por el río Andarax que viene del corredor de las Alpujarras.

Al O de Almería, hasta Aguadulce, la costa está formada por carbonatos triásicos de la unidad de Gádor, del Complejo Alpujárride. Estos carbonatos, aunque localmente tienen pliegues, en su conjunto -con locales excepciones- buzan hacia el S. Dan escarpes del orden de 100-150 m cayendo directamente sobre el mar.

Más al O, el escarpe del borde S de la Sierra de Gádor se prolonga unos $36 \mathrm{~km}$, pero no da actualmente a la costa, sino al Campo de Dalías, un área prácticamente llana, ahora ocupada por una inmensidad de cultivos de plástico. El escarpe está ligeramente retrasado hacia el $\mathrm{N}(\sim 0,75 \mathrm{~km})$ con respecto al tramo de la costa antes descrito.

En ese sector, los materiales triásicos de la Sierra de Gádor situados en el borde $\mathrm{N}$ del escarpe no corresponden todos a la misma altura estratigráfica. En su parte oriental, entre Almería y Aguadulce, afloran términos bajos, calizas más o menos marmorizadas, relativamente masivas, mientras que hacia el $\mathrm{O}$ aparecen progresivamente términos más altos, en los que hay alternancias de calizas, margocalizas, calcosquistos, e incluso localmente algunos términos intercalados que pueden calificarse de filitas (toda la serie presenta un grado bajo a muy bajo de metamorfismo). Además de esos términos, al O de Aguadulce hay también una unidad alpujárride (unidad de Felix) cabalgante sobre la de Gádor, cuya parte meridional visible termina en el escarpe general. Está formada por filitas y carbonatos triásicos de las secuencias inferiores.

Es decir, este escarpe, aunque en su conjunto es rectilíneo, no coincide con una determinada altura estratigráfica de la unidad de Gádor. Los términos de esta unidad van cambiando oblicuamente de $\mathrm{E}$ a O de manera que en conjunto son más modernos los situados en la parte más occidental.

Discordantes sobre ambas unidades existen restos de sedimentos del Mioceno superior dispuestos a modo de tablas que buzan hacia el $\mathrm{S}$ unos $15-20^{\circ}$. Es de interés indicar que al $\mathrm{O}$ de Aguadulce, al $\mathrm{S}$ de la unidad de Felix, hay conglomerados del Mioceno superior con rocas volcánicas (Baena y Ewert, 1983), andesitas, (conocidas también por sondeos, Marín-Lechado, 2005), que parecen distribuidas en dirección E-O.

El escarpe separa dos áreas de muy distinto relieve. Mientras que en su parte $\mathrm{N}$ el relieve es fuerte, superando los 2200 m s.n.m. en la cumbre más alta de la sierra de Gádor, en el S, propiamente en el Campo de Dalías, el relieve es muy suave, con alturas generalmente inferiores a 100 m s.n.m., hasta la línea de costa. Esta parte meridional está formada sobre todo por sedimentos pliocenos y cuaternarios y se encuentra afectada por numerosas fallas, entre las que destacan las de dirección ONO-ESE y NO-SE (Baena y Ewert, 1983; Rodríguez Fernández y Martín Penela, 1993; Marín Lechado, 2005). El borde occidental del Campo de Dalías coincide en gran medida con la falla de Balanegra que es activa (Marín-Lechado, 2005, Gil et al., 2019).

Para Rodríguez Fernández y Martín Penela (1993) el escarpe antes descrito coincide con una falla importante, mientras que para Marín Lechado (2005) este accidente no existe dado que datos geofísicos muestran la continuidad del basamento alpujárride al sur del escarpe. La presencia de materiales del basamento está corroborada en la zona de Guardias Viejas por la existencia de dos pequeños afloramientos del Complejo Alpujárride.

Al O de la Sierra de Gádor el escarpe se interrumpe al ser cortado por el valle del río Adra, pero pasado el valle se recupera y continúa con pocas irregularidades hasta la localidad de Castell de Ferro. Desde Adra forma una costa elevada que asciende con un fuerte gradiente (a poca distancia de la costa se alcanzan en muchos sitios alturas superiores a 300 m s.n.m.). Antes del río Adra, al E de su valle, termina la unidad de Gádor y desde ahí hacia el O son unidades alpujárrides medias y superiores las que aparecen en la costa, es decir, situadas tectónicamente sobre la unidad de Gádor. Los materiales allí aflorantes corresponden fundamentalmente a esquistos, sobre todo esquistos oscuros. Y el buzamiento es hacia el S, con valores generalmente mayores a $30^{\circ}$ o de ese orden.

Directamente al $\mathrm{O}$ de Castell de Ferro hasta Calahonda (unos $5 \mathrm{~km}$ ), la costa está formada por mármoles de una unidad alpujárride media, con espesores superiores a $300 \mathrm{~m}$. Y debido a esto, allí la costa se sitúa aproximadamente $1,5 \mathrm{~km}$ hacia el $\mathrm{S}$ con respecto al escarpe que viene desde Adra. Es decir, los mármoles han sido más resistentes a la erosión que los esquistos y la costa se ha retrasado menos hacia el N. El buzamiento de los mármoles, aunque variable, es generalmente hacia el S. La costa es escarpada con alturas que superan los $300 \mathrm{~m}$ s.n.m. a poca distancia de la línea litoral. 


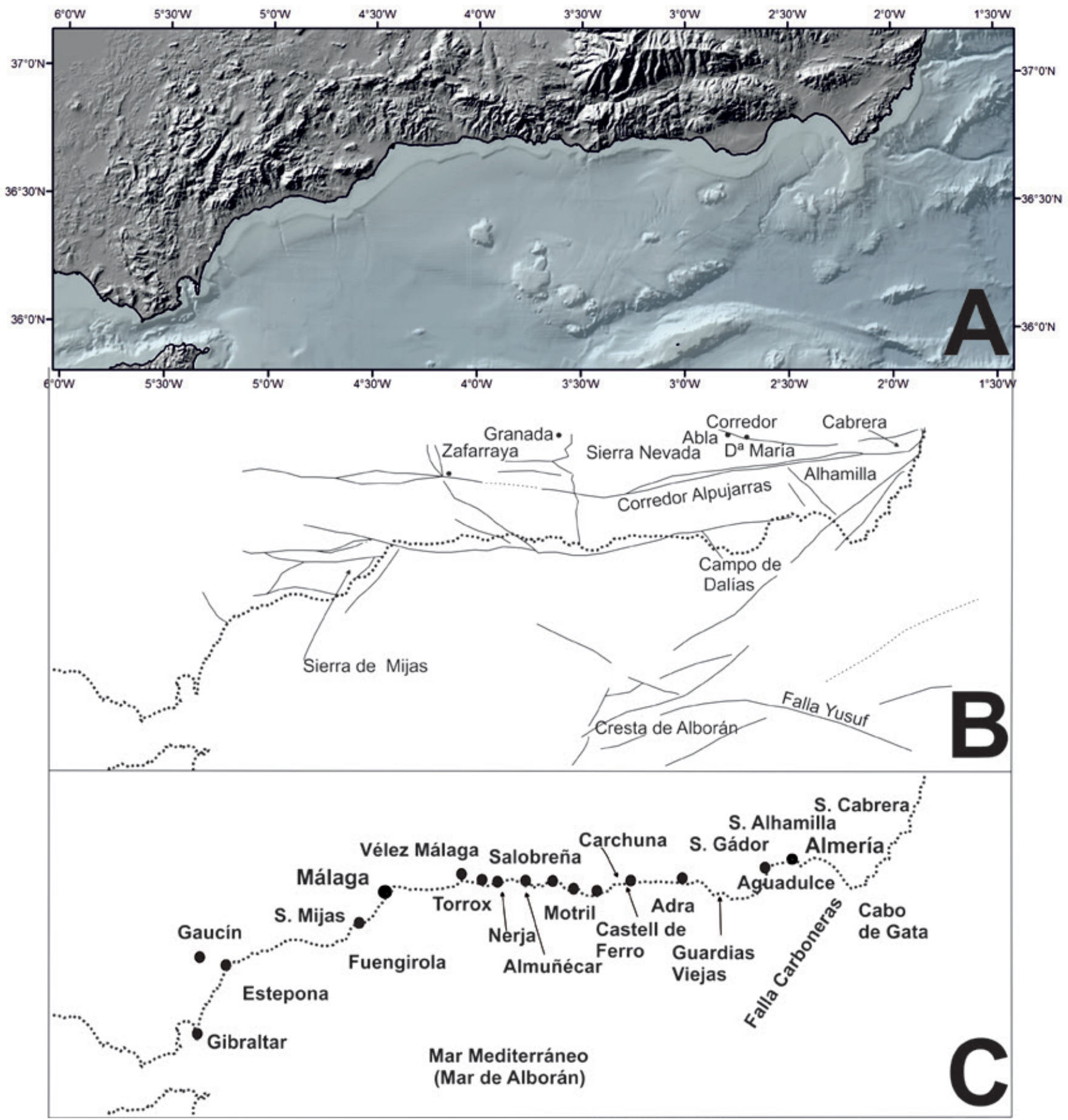

Figura 2. A. Modelo digital del terreno correspondiente a la costa E-O de Andalucía. B. Posición de las principales fallas existentes en este sector. C: Posición de localidades y sierras citadas en el texto. Alguna se cita también en las Figs. 3 y 4.

Figure 2. Digital model of the region of the E-W Andalusian coast. B. Position of the main faults existing in this sector. C: Position of the localities and mountains cited in the text. Some are also indicated in Figures 3 and 4. 
Esquistos de una unidad superior aparecen más al O, en la zona del Cabo Sacratif, directamente tras la Ilanura aluvial de Calahonda-Carchuna, e inmediatamente al $\mathrm{O}$ se encuentra la llanura aluvial de la desembocadura del río Guadalfeo, donde se sitúan Motril y Salobreña.

A partir de Salobreña y hasta Málaga, la costa sigue siendo en su conjunto de dirección E-O, aunque con irregularidades locales. Desde Salobreña a Torrox, las unidades que alcanzan la costa son alpujárrides medias y superiores que presentan en muchos puntos esquistos generalmente oscuros, pero en parte de ese tramo, así entre Almuñécar y Nerja, la costa está formada por mármoles y es muy alta. En su conjunto, los materiales buzan hacia el S. En Nerja hay sedimentos marinos pliocenos emergidos, testimonio de levantamientos costeros (Guerra et al., 2004) relativamente recientes.

Desde Torrox hacia el $\mathrm{O}$, la costa se eleva menos bruscamente. Esto es debido a que ha cambiado la litología. Aparte de la llanura aluvial existente en la zona de Vélez-Málaga, los materiales corresponden a esquistos y lutitas que pertenecen al Complejo Maláguide tectónicamente situado sobre el Complejo Alpujárride. Sus materiales son generalmente menos competentes, más friables.

En resumen, las unidades existentes a lo largo de la costa desde Almería hasta Málaga son, en general, progresivamente más altas tectónicamente hacia el $\mathrm{O}$, aunque con alguna recurrencia.

En Málaga este escarpe continúa tierra adentro unos $32 \mathrm{~km}$, con la misma dirección E-O, alejándose de la costa que ha girado notoriamente, tomando a partir de Málaga una dirección NE-SO.

\section{La prolongación del escarpe al $O$ de Málaga, en la parte occidental de la Zona Interna Bética}

En la parte $\mathrm{N}$ de la prolongación del escarpe (Fig. 4) afloran materiales del Complejo Maláguide. En su borde $\mathrm{S}$ se encuentran materiales del relleno sedimentario de la cuenca de Málaga, sobre todo del Mioceno superior al Cuaternario, además de unidades del Campo de Gibraltar (unidades de los Flyschs) retrocabalgadas sobre la Zona Interna. Más al $\mathrm{O}$, este escarpe se pierde por completo, y el relieve de las sierras de las Nieves y Blanquilla (que en gran medida están formadas por unidades de la Dorsal, además de otras de los complejos Alpujárride y Maláguide) cierra la cuenca de Málaga.

No es ese escarpe el único rasgo importante de dirección E-O en esta zona. Directamente al S existen (Fig. 4) varias fallas dextrorsas de parecida dirección. Una de ellas, dividida en dos ramas, pasa por las localidades de Coín y Cártama y hacia el E se une con la posición del escarpe E-O. La zona situada entre las dos ramas se eleva y forma la Sierra de Cártama (un área aislada dentro de la cuenca de Málaga) y también la pequeña Sierra Gorda de Coín, situada al O de la anterior.

Más al S existe una falla dextrorsa que pasa por el $\mathrm{N}$ de la Sierra de Mijas, la cual hacia el O se desdobla en dos líneas. Una de ellas es la falla del Albornoque (Tubía, 1988) que gira hacia el SO y pasa por el borde $\mathrm{N}$ de Sierra Blanca y separa sus mármoles de otros materiales metapelíticos. La otra continúa directamente hacia el $\mathrm{O}$ y es solo visible en algunos tramos, pues cuando pasa entre materiales metapelíticos o entre las peridotitas es difícil seguir su traza. Alguna de estas fallas tienen un recorrido visible de casi $60 \mathrm{~km} \mathrm{y}$ dan localmente escarpes superiores a $500 \mathrm{~m}$.

Más al S aún, cerca de la costa, entre Estepona y el E de Marbella, los materiales de los complejos Alpujárride y Maláguide son cortados por fallas de apariencia normal que en su conjunto tienen una dirección aproximada E-O (Fig. 4).

Finalmente, hacia el SO, en el límite más occidental entre la Zona Interna y la Externa, se encuentra la falla de Gaucín-Estepona, de dirección NO-SE a ONO-ESE y carácter sinistrorso.

Al $\mathrm{N}$ de esta zona occidental, en el límite entre la Zona Interna y la Externa, existen también fallas dextrorsas E-O (al S de Antequera en la Fig. 4). Estas fallas son en realidad la continuación hacia el $O$ de las que se observan desde la zona más oriental, en el área de Almería (Sierra Alhamilla), pasan por el corredor de las Alpujarras (Sanz de Galdeano et al., 1985) (Figs. 1 a 3) y más adelante, al SO de la cuenca de Granada, se sitúan en el contacto entre la Zona Interna y la Externa.

\section{Algunos datos de la parte sumergida de la costa E-O}

Al S de esta costa, la plataforma marina es estrecha, generalmente del orden de 6 a $8 \mathrm{~km}$, aunque con una anchura inferior a $4 \mathrm{~km}$ en algún sitio. Su estratigrafía y la de áreas marinas adyacentes ha sido estudiadas por Hernández Molina et al. (2002), quienes describen detalladamente las secuencias pliocenas y cuaternarias, pero el significado del talud de la plataforma y si hay fallas y de qué tipo que puedan ser permanece poco claro. En general, se señala la posición del talud sin más interpretación; en algunos perfiles sísmicos se marcan fallas lístricas en los sedimentos.

En el artículo de Ballesteros et al. (2008) se señala la presencia de una gran falla transcurrente dextrorsa en la base del talud, la cual está cortada por varias fallas transversales de dirección NO-SE (también dextrorsas) que la desplazan (véanse sus figuras 5 y 21 y la Fig. 1 


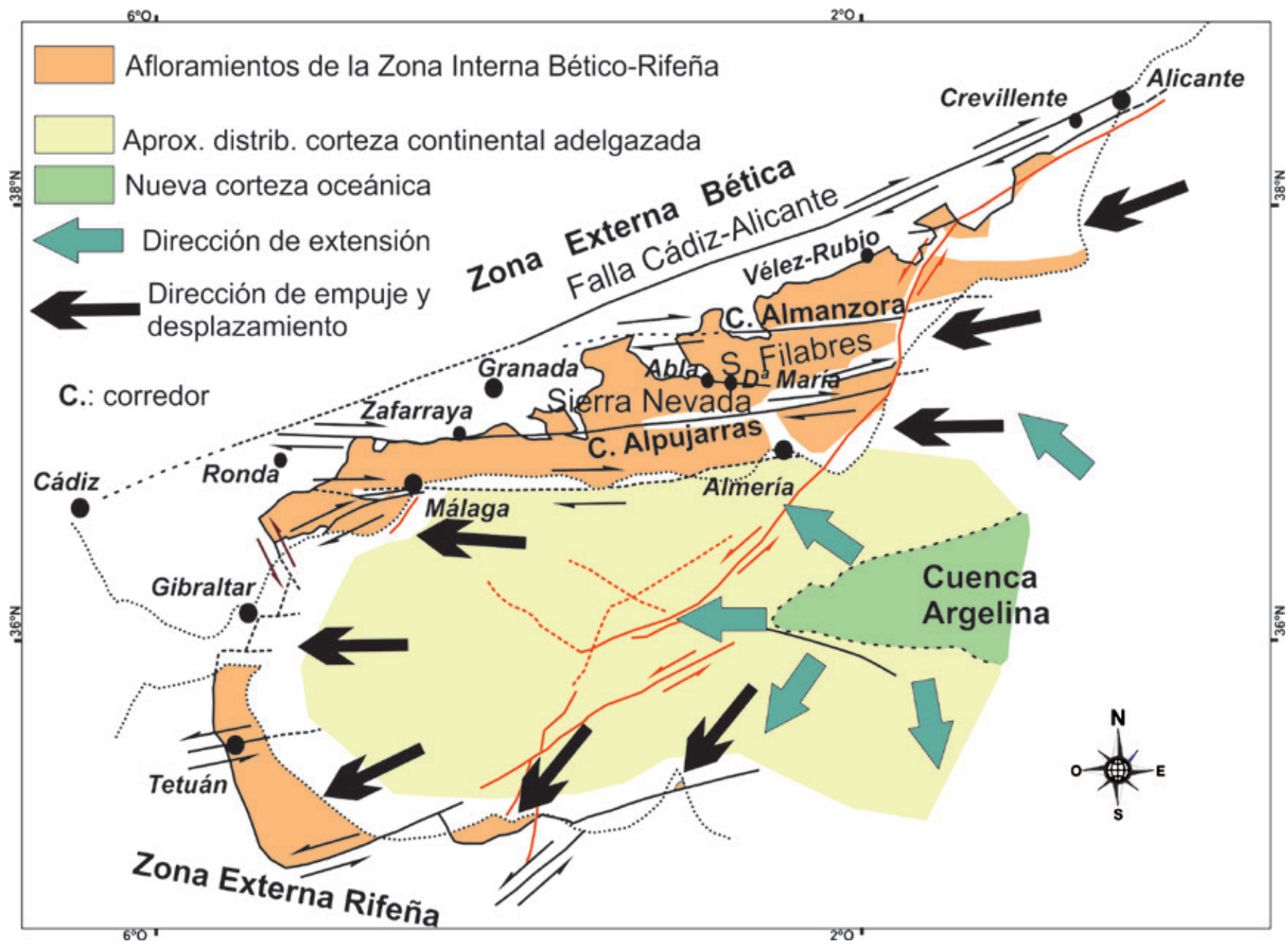

Figura 3. Esquema del conjunto de sectores en que la Zona Interna Bético-Rifeña se encuentra dividida. En rojo se marcan fallas posteriores, particularmente las que cruzan el mar de Alborán. Las flechas negras grandes indican las direcciones generales de desplazamiento. Modificado de Sanz de Galdeano (1996).

Figure 3. Scheme of the main segments in which the Betic-Rifian Internal Zone is divided. In red later faults are marked, particularly those crossing the Alboran Sea. Modified from Sanz de Galdeano (1996).

B en el presente artículo, donde se dibuja en línea roja a puntos). Por tanto, los movimientos de estas fallas transversales son posteriores a esa gran falla dextrorsa del borde sur de la plataforma. Esta falla descrita por Ballesteros et al. (2008) cuando llega hacia el O a Málaga adapta su dirección a la de la costa en el tramo Fuengirola-Torremolinos, es decir NNE-SSO. También estos autores la adaptan a lo largo del talud en la costa del Campo de Dalías. Para esta interpretación no dan datos, aunque citan varios artículos como fuentes, pero la interpretación parece propia de ellos.

La plataforma tiene algunas irregularidades, en especial en el Campo de Dalías, que en principio “debería" ser una zona sumergida. Las fallas antes citadas allí son las responsables de su emersión (alguna de esas fallas es aún activa, Marín-Lechado et al., 2004; Marín-Lechado, 2005). Es un rasgo formado a partir del Plioceno, superpuesto a la dirección E-O de la costa. En ese aspecto, el Campo de Dalías forma parte de la plataforma, pero que allí está emergida y es más ancha que en otras zonas.

\section{Discusión}

\section{Sobre las unidades tectónicas que aparecen a lo lar- go de la costa}

De su análisis se deducen dos hechos:

a) Esta costa no coincide con una determinada unidad tectónica, ni siquiera con un determinado comple- 
jo tectónico. A lo largo de ella son distintas unidades alpujárrides y el Complejo Maláguide los que afloran. Es decir, los contactos entre las distintas unidades tectónicas aparecen de forma oblicua en esta costa. No se mantiene una determinada posición tectónica.

b) Existe una neta tendencia a que las unidades situadas en posiciones tectónicas superiores aparezcan hacia el $\mathrm{O}$, mientras que las inferiores lo hacen hacia el E. Es decir, en la parte occidental (en la cuenca de Málaga) es el Complejo Maláguide el que se encuentra en el borde del escarpe, lo que continúa al E en la costa hasta las proximidades de Vélez-Málaga. Más al E son las unidades altas del Complejo Alpujárride (Fig. 1 B) y, conforme se avanza hacia Almería, son las unidades medias e inferiores alpujárrides las que van apareciendo progresivamente. Esta distribución no es lineal, sino que a veces la superposición de fallas transversales a la costa y alguna estructura de plegamiento hacen que alternen las unidades medias y las inferiores. Pero en su conjunto esa es la tendencia.

Lo anterior muestra algo que suele pasar desapercibido: la Cordillera Bética en su conjunto está más levantada hacia el $E, y$, al contrario, se hunde más al O. Así, el complejo tectónico inferior de la Cordillera, el Nevado-Filábride, y las unidades inferiores del Alpujárride afloran en su parte oriental, mientras que en la occidental lo hacen las unidades del Campo de Gibraltar, la Dorsal, el Complejo Maláguide y las unidades superiores del Alpujárride. Esto es congruente con la interpretación de que en el proceso de extensión y apertura del mar de Alborán, además que los materiales de la Zona Interna fueran empujados sobre todo hacia el $\mathrm{O}$, se fueron levantando sus bordes, de manera que se elevaron más en su parte $E$, donde la apertura de este mar fue mayor.

\section{Sobre las fallas de la parte occidental de la Zona In- terna Bética y los sectores en que esta se divide}

En la parte occidental de la Zona Interna Bética, las grandes fallas E-O dextrorsas antes señaladas desplazan hacia el $\mathrm{O}$ el sector más meridional de dicha zona (Figs. 2, 3 y 4). Este desplazamiento es del orden de 40 $\mathrm{km}$ si se compara su posición con la del sector situado al $\mathrm{N}$ de Málaga. A su vez, en el extremo occidental, la falla NO-SE de Gaucín-Estepona, sinistrorsa y conjugada con las anteriores, también contribuye al avance de ese sector de la Zona Interna.

Esta división en sectores de la Zona Interna no es un fenómeno local. De hecho toda la Zona Interna está segmentada de esa forma formando corredores. De ellos los mejor definidos son los del Almanzora y de las Alpujarras (Fig. 3). También las sierras Nevada y Fi- labres están separadas por una falla E-O que hacia el E no se ve seguir (su existencia se deduce por el desplazamiento vertical, superior a $1000 \mathrm{~m}$, que muestran términos de su serie litológica entre las localidades de Abla y $D^{a}$ María, Fig. 3). Sin embargo, aún más al $E$, ya cerca de la costa, por el $\mathrm{N}$ de Sierra Cabrera, existe una falla importante dextrorsa que está en su continuidad y es de igual dirección.

En cada caso, el sector meridional situado directamente al $\mathrm{S}$ de cada corredor ha avanzado más hacia el $\mathrm{O}$ que el que se encuentra al N, coadyuvando así al desplazamiento de la Zona Interna.

Además de los anteriores sectores, en el contacto entre la Zona Interna y la Externa se encuentra el corredor de Vélez-Rubio (en la provincia de Almería) de dirección aproximada N70 ${ }^{\circ} \mathrm{E}$. Y entre las provincias de Granada y Málaga (entre Zafarraya y el NE de Ronda) (Fig. 3) este mismo contacto, es rectilíneo, de dirección E-O, aunque no forma un corredor deprimido, al contrario, forma una larga cresta. Otros segmentos de este contacto no tienen esa dirección aproximada E-O. Es decir, dicho contacto muestra irregularidades cuyos segmentos E-O, o de parecida dirección, indican desplazamientos hacia el $\mathrm{O}$ de los sectores más meridionales.

$\mathrm{Y}$ a los anteriores rasgos E-O hay que añadir la costa ahora estudiada, con una orientación similar.

\section{Sobre algunas características de la costa E-O}

En el borde $S$ de la Sierra de Gádor. Según Marín Lechado (2005) entre la Sierra de Gádor y la llanura del Campo de Dalías no existe falla alguna, pues datos gravimétricos muestran la continuidad del basamento alpujárride al sur del escarpe. Pero esa continuidad captada por gravimetría, sin duda cierta, no necesariamente implica que no haya falla por dos razones: la falla puede ser fundamentalmente de salto en dirección, lo que no precisa un salto vertical que es lo que se observaría por gravimetría, $y$, además, el escarpe en este borde S de la Sierra de Gádor está algo retrasado con respecto al existente entre Almería y Aguadulce. Es decir, la falla en realidad puede situarse algo más al $\mathrm{S}$, incluso más de 1 o 2 kilómetros.

A favor de la existencia de la falla, aunque no sea un dato concluyente, está el corte oblicuo de las formaciones litológicas que encaja bien con la existencia de una falla E-O. También, las andesitas antes citadas, al parecer con una distribución E-O, que necesitan para su salida al menos de una fisura profunda que atraviese la corteza.

En el resto de la costa E-O. Desde el Campo de Dalías hasta Málaga, dada la poca anchura que tiene la 


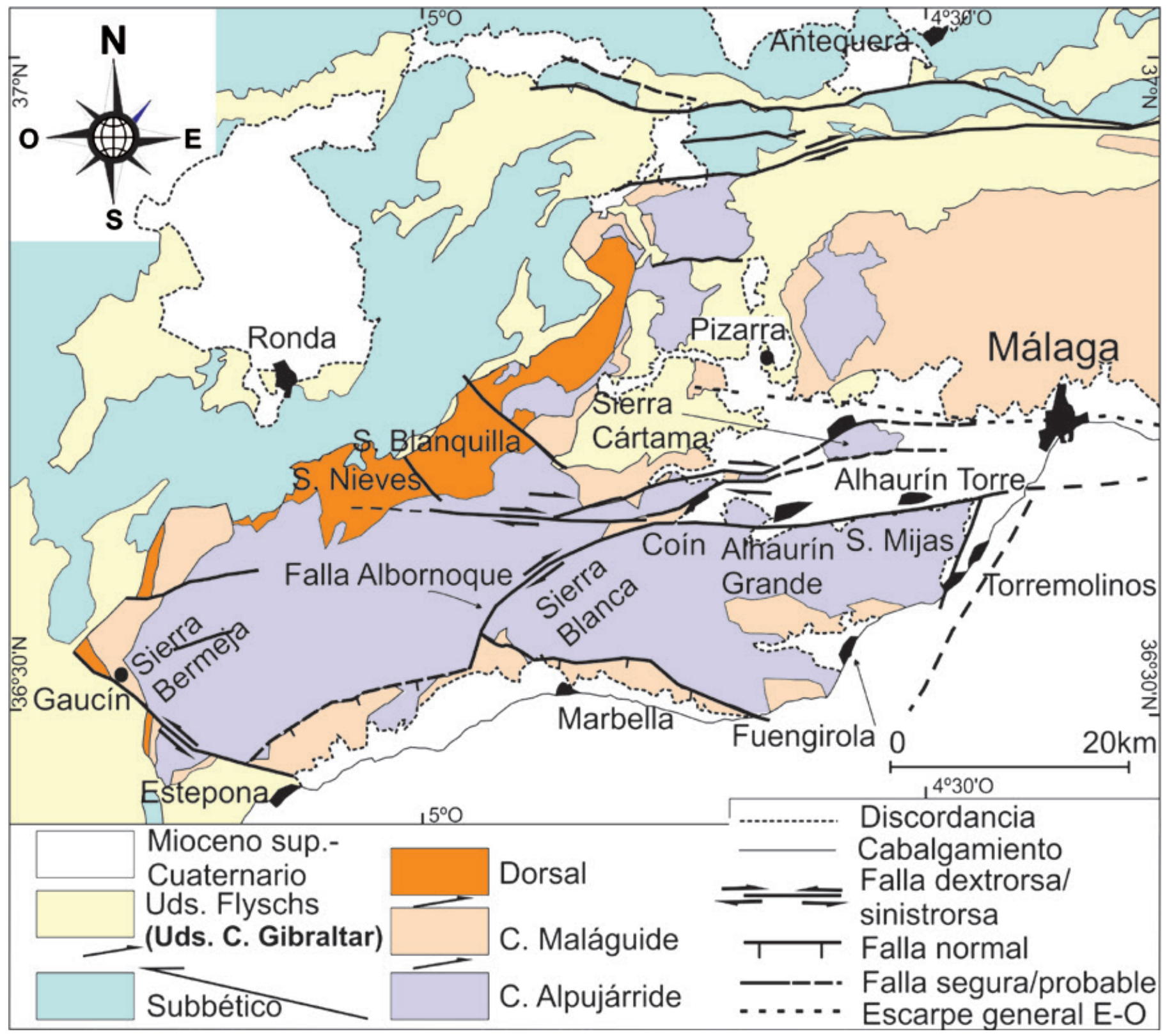

Figura 4. Esquema geológico de la parte occidental de la Zona Interna, incluyendo sobre todo las fallas E-O o próximas a esta dirección. Su posición se indica en B de la Fig. 1.

Figure 4. Geological scheme of the western part of the Internal Zone, including especially the E-W faults or those close to this direction. Its position is indicated in B of Figure 1.

plataforma marina, no existe espacio para absorber la oblicuidad que muestran las unidades tectónicas en su distribución en la parte emergida (Fig. 1 B). La interpretación más fácil es que se trata de un corte producido por una falla que se situaría en el borde de la plataforma. Según esto, la costa actual correspondería al escarpe retrasado de la falla, menos retrasado allí donde las rocas son más resistentes a la erosión, como por ejemplo donde existen mármoles o esquistos con muchas cuarcitas.
La falla de salto en dirección que muestran Ballesteros et al. (2008) no la justifican. En ella, no parece aceptable que siga siempre el límite de la plataforma en todo su recorrido, como ocurre -según señalan- en el tramo de Málaga a Fuengirola donde toma una dirección NNE-SSO, y en el Campo de Dalías, Pero esos dos sectores sí pueden ser controlados por otras faIlas. Así, en el sector de Málaga a Fuengirola, el borde oriental de la Sierra de Mijas corresponde a una importante falla de dirección NNE-SSO (Fig. 4) que 
la corta bruscamente. $Y$ en el mar puede haber otras de parecidas direcciones. Por su parte, el borde occidental del Campo de Dalías coincide con la citada falla activa de Balanegra, mientras que no conocemos qué controla el borde oriental.

Es decir, en esos sectores, los de Málaga-Fuengirola y del Campo de Dalías, también parecen haber fallas. De este modo, la una única falla que indican Ballesteros et al. (2008) en realidad corresponde a varias fallas. Pero en su parte central, la interpretación de dichos autores de que sea una falla dextrorsa coincide con la que aquí se propone.

\section{Interpretación general}

La interpretación del significado de la costa E-0 hay que encuadrarla en el conjunto de la propia evolución de la Cordillera Bética, incluyendo el mar de Alborán y el $\mathrm{N}$ del Rif.

Durante la formación de la cuenca Argelina (o Argelo-Provenzal), en su parte occidental se abrió el mar de Alborán en un importante proceso de extensión y adelgazamiento cortical. Este proceso afectó a la periferia, a los bordes adyacentes de la Cordillera Bética y del Rif. Por ello, toda la Zona Interna, además de lo que de ella quedaba adelgazada dentro del propio mar de Alborán, fue progresivamente expulsada hacia el O (al SO en el Rif y al O y ONO en las Béticas). A la vez, la parte oriental de la Zona Interna Bética fue más levantada que la occidental, relativamente hundida.

En esta expulsión la Zona Interna Bética fue segmentada (Fig. 3), de manera que los sectores más meridionales son los que se desplazaron más al O. Por ello, su parte más occidental fue la más desplazada (es el área correspondiente a la figura 4).

Las fallas que facilitaron esta transcurrencia son fundamentalmente las situadas en los corredores antes citados. El propio contacto entre las Zonas Interna y Externa responde a este mecanismo fundamentalmente transcurrente (a causa de la colisión oblicua de la Zona Interna, la Zona Externa fue muy deformada y, además de cabalgamientos, se formó en ella una larga banda de fracturas, la falla de Cádiz-Alicante (Foucault, 1974; Sanz de Galdeano, 1983, 2008 b) (Fig. 3).

Es en este proceso de segmentación donde se sitúa la propuesta falla E-O que conforma la costa de Málaga a Almería. Fue, en la presente interpretación, la falla (o quizás mejor fallas en algún caso) más meridional de dirección E-O que afectó a la Zona Interna Bética, y la que produjo, dada su posición, mayor desplazamiento hacia el $\mathrm{O}$ en dicha zona. El valor de ese desplazamiento deducido a partir de las posiciones del frente de la Zona Interna Bética en los sectores del
Ny del S de Málaga es del orden de $40 \mathrm{~km}$. Pero, si se quiere obtener un valor mínimo de desplazamiento de la Zona Interna Bética hacia el $\mathrm{O}$, hay que ir sumando los producidos por las otras fallas situadas en los corredores.

Las diferentes fallas citadas que dividieron en sectores a la Zona Interna constituyeron desde entonces zonas de debilidad. Por ello, cuando a partir de finales del Mioceno medio, ya prácticamente en el superior, se instauró en la región una compresión aproximadamente NNO-SSE a N-S, la posición de esas fallas determinó la localización de los principales pliegues de la Zona Interna, superpuestos a la tectónica de mantos de corrimiento. Es decir, los sectores situados entre esas fallas se plegaron formando grandes antiformes. De ellos destacan el de Sierra Nevada y también el de la Sierra de los Filabres. Igualmente, más al $\mathrm{N}$ la alineación de la Sierra de las Estancias en su conjunto tiene una disposición antiforme y lo mismo puede decirse más al $\mathrm{S}$, en todo el alineamiento que desde los Montes de Málaga hacia el E llega a la Sierra de Gádor y continúa hasta el mar por las sierras Alhamilla y Cabrera. Es decir, directamente al $\mathrm{N}$ de la costa E-O existe también una disposición antiforme. Por el contrario, los sinformes se sitúan sobre las zonas de fallas que dividieron la Zona Interna en sectores.

Significa lo anterior que el buzamiento hacia el S en la costa $\mathrm{E}-\mathrm{O}$ se produjo fundamentalmente cuando se formó el citado antiforme. Fue por tanto a partir del Mioceno superior, ya avanzado, cuando se creó el relieve del antiforme (aunque la falla propuesta se formara previamente). Entonces comenzó la erosión de ese flanco sur del antiforme, formándose el escarpe que fue progresivamente retrasado hacia el N, a la vez que se incrementaba su altura.

En consecuencia, la costa E-O actual se ha producido por una combinación de dos causas. La primera es que se formó una importante falla E-O transcurrente dextrorsa que se sitúa en la base del talud. La segunda es que, tras formarse el antiforme que existe directamente al $\mathrm{N}$, comenzó a erosionarse su flanco sur, formándose progresivamente el escarpe, en general más alto cuanto más se ha retrasado hacia el $\mathrm{N}$, pues afecta a partes más altas de ese flanco.

Hay que citar que a partir del paso del Mioceno medio al superior se formaron varias de las fallas transversales a la costa $\mathrm{E}-\mathrm{O}$, antes citadas, y que, naturalmente han afectado a la falla $\mathrm{E}-\mathrm{O}$ propuesta. Algunas de ellas muy importantes, tal como la falla de Carboneras, que tiene ligado el volcanismo de Cabo de Gata.

Finalmente, conviene indicar que aunque se propone la existencia de una falla E-O situada a lo largo de la costa, más bien en la posición del talud, probablemente la realidad sea algo más compleja y existan, 
además, otras fallas de menor importancia que añadirían alguna complejidad al cuadro aquí presentado.

\section{Conclusiones}

En la costa E-O del sur de España, situada en la Zona Interna Bética, las unidades tectónicas se disponen oblicuamente, de manera que, en general, las inferiores afloran en la parte oriental y las más altas aparecen en la parte más occidental. Esta oblicuidad que no mantiene un nivel estructural determinado parece no tener espacio para recuperarse antes del talud de la plataforma continental que en la mayor parte de los puntos es estrecha, del orden de 6 a $8 \mathrm{~km}$. Esta disposición sugiere que a lo largo de este talud existe una falla importante dextrorsa. Esto no está necesariamente en contradicción con el hecho de que en la parte S de la Sierra de Gádor no se observe por gravimetría la presencia de una falla pues con esta técnica los desplazamientos horizontales no se captan, además de que intentar verla en la posición actual del escarpe no es acertado, pues en general el escarpe está retrasado hacia el $\mathrm{N}$, incluso varios kilómetros, según los sectores.

La dirección de esta costa coincide con la de importantes fallas E-O que cortaron la Zona Interna y que facilitaron el desplazamiento hacia el $\mathrm{O}$ de los distintos sectores en que la dividieron. Este parece ser también el caso de la propuesta falla de la costa E-O. De ella, solo en la parte occidental emergida de la Zona Interna Bética se observan importantes fallas dextrorsas que desplazan dicha zona del orden de $40 \mathrm{~km}$. El resto está sumergido.

La formación de estas fallas estuvo ligada con la apertura del mar de Alborán (extremo occidental de la cuenca Argelina), en cuyo proceso de extensión su borde N, ocupado por la Zona Interna Bética, fue fuertemente empujado, segmentado y desplazado hacia el $\mathrm{O}$, tanto más cuanto más cercano se encontraba cada sector al eje de expansión de Alborán.

Pero la formación del escarpe se liga al posterior proceso de creación de antiformes en la Zona Interna, en particular al situado directamente al $\mathrm{N}$ de la costa $\mathrm{E}-\mathrm{O}$. Conforme se fue erosionando su flanco sur, a partir de la propuesta falla $\mathrm{E}-\mathrm{O}$, fue creándose el escarpe, en general más alto cuanto más ha avanzado la erosión.

\section{Agradecimientos}

Agradezco las correcciones y sugerencias, incluyendo la de introducir una nueva figura, a los revisores J.J. Durán y F. Bohoyo del IGME, así como las de otro revisor anónimo. Igualmente al Editor, E. Pardo, por su ayuda y paciencia. Todas ellas han contribuido claramente a la mejora del artículo. Es una contribución del proyecto DAMAGE (AEI/FEDER CGL2016-80687-R) de la DGICYT, y del grupo de investigación RMN-370 de la Junta de Andalucía.

\section{Referencias}

Baena, J. y Ewert, K. 1983. Mapa geológico nº 1058 (Roquetas de Mar).1:50.000. Mapa geológico de España (1:50.000), Instituto Geológico y Minero de España, plan MAGNA, $34 \mathrm{p}$.

Ballesteros, M., Rivera, J., Muñoz, A., Muñoz-Martín, A., Acosta, J., Carbó, A. y Uchupi, E. 2008. Alboran Basin, southern Spain-Part II: Neogene tectonic implications for the orogenic float model. Marine and Petroleum Geology, 25, 75-101.

Boillot, G., Montadert, L., Lemoine, M. y Biju-Duval, B. 1984. Les marges continentales actuelles et fossiles autour de la France. Ed. Masson, Paris, 342 p.

Durand-Delga, M. y Fontboté, J.M. 1980. Le cadre structural de la Méditerranée occidentale. 26 Congrès. Géol. Intern., Paris. Les Chaînes alpines issues de la Téthys. Mém. B.R.G.M., 115, 67-85.

El Kadiri, K., Sanz de Galdeano, C. Pedrera, A., Chalouan, A., Galindo-Zaldívar, J., Julià, R., Akil, M., Hlila, R. y Ahmamou, M. 2010. Eustatic and tectonic controls on Quaternary Ras Leona marine terraces (Strait of Gibraltar, northern Morocco). Quaternary Research, 74, 277-288.

Foucault, A. 1974. Travaux et titles scientifiques et universitaires. Paris, $36 \mathrm{p}$.

Gil A.J., Sánchez-Alzola A., Galindo-Zaldívar J., Borque M.J., Lacy M.C., Avilés M., Alfaro P., López Garrido A.C., Sanz de Galdeano C., Herrera A., Chacón F., Madrigal M., Blanca S., Moreno J.C. y Tendero V. 2019. Estimation of the crustal velocity field in the Balanegra fault from GPS position time series in 2006 - 2018. Proceedings ITISE-(International Conference on Time Series and Forecasting). Granada, 25-27 septiembre, 790-796. I.S.B.N: 978-8417970-78-9

Gómez de la Peña, L., Ranero, C. R. y Gràcia, E. 2018. The crustal domains of the Alboran Basin (western Mediterranean). Tectonics, 37, 3352-3377. https://doi.or$\mathrm{g} / 10.1029 / 2017$ TC004946

Grevemeyer, I., Gràcia, E., Villaseñor, A., Leuchters, W. y Watts, A.B. 2015. Seismicity and active tectonics in the Alboran Sea,Western Mediterranean: Constraints from an offshore-onshore seismological network and swath bathymetry data. J. Geophys. Res. Solid Earth, 120, 83488365. http://dx.doi.org/10.1002/2015JB012073.

Groupe de recherche néotectonique de I'Arc deGibraltar. 1977. L'histoire tectonique récente (Tortonien à Quater- 
naire) de l'Arc de Gibraltar et des bordures de la mer d'Alboran. Bulletin de la Societé Géologique de France, 19 (3), 575-614.

Guerra Merchán, A., Serrano, F. y Ramallo, D. 2004. Geomorphic and sedimentary Plio-Pleistocene evolution of the Nerja (northern Alboran Basin, Spain). Geomorphology, 60, 89-105.

Hernández Molina, F.J., Somoza, L., Vázquez, J.T., Lobo, F., Fernández-Puga, M.C., Llave, E. y Díaz-del Río, V. 2002. Quaternary stratigraphic stacking patterns on the continental shelves of the southern Iberian Peninsula: their relationship with global climate and palaeoceanographic changes. Quaternary International, 92, 5-23.

Lario, J., Zazo, C., Somoza, L., Goy, J.L., Hoyos, M., Silva, P.G. y Hernández Molina, F.J. 1993. Los episodios marinos cuaternarios de la costa de Málaga (España). Revista Sociedad Geológica de España, 6, 3/4, 41-46.

Larouzière, F.D. de, Bolze, J., Bordet, P., Hernandez, J., Montenat, Ch. y Ott d'Estevou, Ph. 1988. The Betic segment of the lithosperic Trans-Alboran shear zone during the Late Miocene. Tectonophysics, 152, 41-52.

Marín Lechado, C. 2005. Estructura y evolución tectónica reciente del Campo de Dalías y de Níjar en el contexto del límite meridional de las Cordilleras Béticas Orientales. Tesis Univ. Granada-Instituto Geológico y Minero de España. Granada, 266 pp.

Marín Lechado, C., Galindo-Zaldívar, J., Rodríguez-Fernández, L.R. y Pedrera, A. 2004. Desarrollo progresivo de pliegues y formación del frente montañoso meridional de la Cordillera Bética en el Campo de Dalías. Geo-Temas, 6(3), 163-166.

Perea, H., Gràcia, E., Martínez-Loriente, S., Bartolome, R., Gómez de la Peña, L., de Mole, B., Moreno, X., Lo lacono, C., Diez, S., Tello, O., Gómez-Ballesteros, M. y Dañobeitia, J.J., 2018. Kinematic analysis of secondary faults within a distributed shear-zone reveals fault linkage and increased seismic hazard. Marine Geology, 399, 23-33.

Pineda Velasco, A., Goy, J.L., Zazo, C. y Giner, J. 1981. Mapa geológico $n^{\circ} 1059$ (Cabo de Gata). Mapa geológico de España (1:50.000), Instituto Geológico y Minero, plan MAGNA, $41 \mathrm{p}$.

Rodríguez-Fernández, J. y Martín-Penela, A. 1993. Neogene evolution of the Campo de Dalias and the surrounding offshore areas - (Northeastern Alboran Sea). Geodinamica Acta, 6, 4, 255-270.

Sanz de Galdeano, C. 1983. Los accidentes y fracturas principales de las Cordilleras Béticas. Estudios Geológicos, 39, 157-165.

Sanz de Galdeano, C. 1990. Geologic evolution of the Betic Cordilleras in the Western Mediterranean, Miocene to the present. Tectonophysics, 172, 107-119.

Sanz de Galdeano, C. 1996. The E-W segments of the contact between the External and Internal Zones of the Betic and Rif Cordilleras and the E-W corridors of the Internal Zone (A combined explanation). Estudios Geológicos, 52, 123-136.

Sanz de Galdeano, C. 2008a. La Cordillera Bética: Una cadena fragmentada. Geo-temas, 10, 413-416.

Sanz de Galdeano, C. 2008b. The Cádiz-Alicante Fault: an important discontinuity in the Betic Cordillera. Revista de la Sociedad Geológica de España, 20, 49-58.

Sanz de Galdeano, C. 2012. Estructuras ligadas al contacto entre las zonas Interna y Externa de la Cordillera Bética al Norte de Málaga. Geo-temas 13. VIII Congreso Geológico de España, Oviedo, 17-19 julio, 449-452.

Sanz de Galdeano, C., Rodríguez Fernández, J. y López Garrido, A.C. 1985. A strikeslip fault corridor within the Alpujarra Mountains (Betic Cordilleras, Spain). Geologische Rundschau. 74- (3), 641-655.

Serrano, F. 1992. Biostratigraphic control of Neogene volcanism in Sierra de Gata (south-east Spain). Geologie en Mijnbouw, 71, 3-14.

Tubía, J.M. 1988. Estructura de los Alpujárrides occidentales: Cinemática y condiciones de emplazamiento de las peridotitas de Ronda. Parte I: Características litológicas. Boletín Geológico y Minero, 99, 2, 165-212.

Woerd, J., Dorbath, C., Ousadou, F., Dorbath, L., Delouis, B., Jacques, E., Tapponnier, P., Hahou, J., Menzhi, M., Frogneux, M. y Haessler, H., 2014. The Al Hoceima Mw 6.4 earthquake of 24 February 2004 and its aftershocks sequence. Journal of Geodynamics, 77, 89-109.

Zazo, C., Goy, J.L., Dabrio, C.J., Bardají, T., Hillaire-Marcel, C., Ghaleb, B., González-Delgado, J.A. y Soler, V., 2003. Pleistocene raised marine terraces of the Spanish Mediterranean and Atlantic coasts: records of coastal uplift, sea-level highstands and climate changes. Marine Geology, 194 (1-2), 103-133.

Recibido: julio 2019

Revisado: noviembre 2019

Aceptado: enero 2020

Publicado: marzo 2021 